\title{
An Automatic Interconnection Rectification Technique for SoC Design Integration
}

\author{
Chun-Yao Wang, Shing-Wu Tung and Jing-Yang Jou \\ Department of Electronics Engineering, National Chiao Tung University \\ Hsinchu 300, Taiwan, R.O.C. \\ Tel: 886-3-5726111, Fax: 886-3-5710580 \\ e-mail: \{wcyao,swtung,jyjou\}@eda.ee.nctu.edu.tw
}

\begin{abstract}
This paper presents an automatic interconnection rectification (AIR) technique to correct the misplaced interconnection occurred in the integration of a SoC design automatically. The experimental results show that the AIR can correct the misplaced interconnection and therefore accelerates the integration verification of a SoC design.
\end{abstract}

\section{INTRODUCTION}

In the SoC era, system level integration and platform-based design [1] are evolving as a new paradigm in system designs, hence, design reuse and reusable building blocks (cores) trading are becoming popular. However, present design methodologies are not enough to deal with cores which come from different design groups and are mixed and matched to create a new system design. In particular, design verification is one of the most difficult task.

The focus of core-based design verification should be on how the cores communicate with each other [2]. However, prior to the interface verification, the interconnection between the cores in a SoC have to be verified first. This is because the SoC integrator has to connect a large number of ports in a SoC design. The likelihood of interconnection misplacements between the cores is high. Thus, the interconnection verification can be conducted as the first step to the interface verification between the cores in a SoC design.

By creating the testbenches at a high level, a connectivitybased design fault model, port order fault (POF), is proposed in [3]. This fault model is similar to the Type $\mathrm{H}$ design error "incorrectly placed wire" in the logic level [4] [5]. The POF-based automatic verification pattern generation (AVPG) are also developed in [6] [7]. The AVPG are effective in generating the verification pattern set for detecting the misplacements of interconnection in a SoC design. However, the diagnosis and correction issues on the misplaced interconnection are even more important for SoC verification. Thus, to accelerate the SoC integration process, this paper presents an automatic interconnection rectification (AIR), which not only detects the erroneous interconnection among the cores, but also diagnoses and corrects them automatically.
Traditional diagnosis and correction algorithms in the logic level can be divided into two categories with respect to the underlying techniques: those based on symbolic techniques [8] [9] and those based on simulation techniques [5] [10] [11]. The approaches based on symbolic techniques can return valid correction and handle circuits with multiple errors well, however, they are not applicable to circuits that have no efficient Ordered Binary Decision Diagram (OBDD) [12] representation. Thus, to verify the interconnection among the IP cores with all description levels (soft, firm, and hard cores) embedded into a system, the AIR algorithm has to deal with IP cores that are described in different levels, for example, logic level, register transfer (RT) level, or even behavioral level. Consequently, the symbolic approach is inadequate to this application and the simulation based AIR algorithm is presented.

\section{PRELIMINARY}

Definition 1: The type I POF is at least an output misplaced with an input. The type II POF is at least two inputs misplaced. The type III POF is at least two outputs misplaced [3].

It has been proven that the type II POFs dominate the other two types of POFs [6]. Thus, the AIR focuses on the type II POFs.

Definition 2:A port sequence is an input port numbers permutation. The fault free port sequence (FFPS) is a port sequence that none of the input ports is misplaced. For an $N$-input core, the $N$ ! permutations represent the $N$ ! port sequences. Except the FFPS, the remaining ( $N$ !-1) port sequences are called faulty port sequences (FPSs).

Example 1: The schematic representation of the FFPS 1234 and the FPS 1423 of BLK2 are shown in Fig. 1(a) and Fig. 1(b), respectively.

The undetected port sequences (UPSs) representation used in the AVPG [6] [7] is to implicitly represent the undetected port sequences remained in the fault set. Since it is also used in the AIR algorithm, we introduce it here briefly.

Example 2: Given an 8-input core, the input ports are numbered from 1 to 8 . The (12345678) represents the UPSs that caused by all possible misplacements among the port numbers 


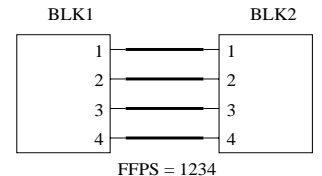

(a)

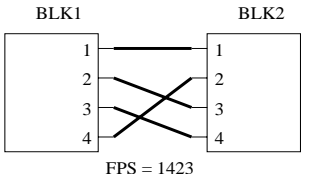

(b)
Fig. 1. The schematic representation of the FPS

in the same group, i.e., port 1 to port 8. The (125)(4)(3678) indicates the UPSs that caused by all possible misplacements among the port numbers 1,2 and 5 and/or all possible misplacements among the port numbers $3,6,7$ and 8 . The $(1)(2)(3)(4)(5)(6)(7)(8)$ represents 8 !-1 POFs are all detected. If the UPSs representation is induced from (12345678) to $(1)(2)(3)(4)(5)(6)(7)(8)$, all POFs are detected.

The environment and mechanism of POF verification, which exploits IEEE P1500 SECT, can be found in [6]. Thus, we omit describing them here due to the page limit.

\section{THE AIR ALGORITHM}

\section{A. AIR Overview}

The input to the AIR is the simulation model of an IP core. The four stages of AIR are pattern generation, fault detection, fault diagnosis, and fault correction as shown in Fig. 2. In addition to these four stages, an instantaneous UPSs representation is associated with the AIR. This UPSs representation can indicate the remaining UPSs currently and guides the generation of further verification patterns. If the UPS is empty, the AIR is terminated and the interconnection in the integrated design are correct.

When a pattern set $P_{i}$ is selected as the verification pattern set, some FPSs will be detected by $P_{i}$ and the UPSs will be reduced from $\Pi_{i-1}$ to $\Pi_{i}$ where $\Pi_{i}$ denotes the remaining UPSs after the verification by $P_{i}$. Then the pattern generation stage generates further verification pattern sets in the next iteration according to $\Pi_{i}$. Each $P_{i}$ corresponds to a set of FPSs and is responsible for detecting them. The FPSs which are detected by $P_{i}$ are denoted as $\operatorname{FPSs}\left(P_{i}\right)$. The relationship of $P_{i}, \operatorname{FPSs}\left(P_{i}\right)$, and UPSs $\Pi_{i}$ is shown in Fig. 3. In Fig. 3, the initial UPSs are denoted as $\Pi_{0}$ and we assume the pattern set $P_{i-1}$ is generated before $P_{i}$. When the last pattern set $P_{t}$ is generated, the UPS is $\Pi_{t}$ and it is the FFPS.

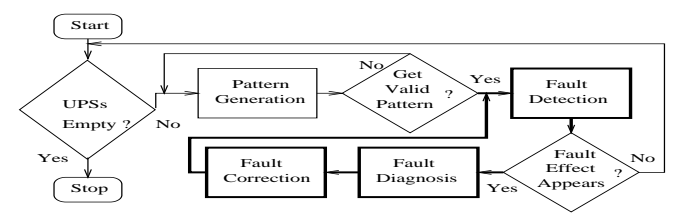

Fig. 2. The AIR flow

\begin{tabular}{|c|c|c|}
\hline $\begin{array}{c}\text { pattern } \\
\text { set }\end{array}$ & detected FPSs & $\begin{array}{c}\text { remaining } \\
\text { UPSs }\end{array}$ \\
\hline- & - & $\Pi_{0}=$ ini. UPS \\
\hline$P_{1}$ & FPSs $\left(\mathbf{P}_{1}\right)$ & $\Pi_{1}$ \\
\hline$P_{2}$ & FPSs $\left(\mathbf{P}_{2}\right)$ & $\Pi_{2}$ \\
\hline $\mathbf{P}_{3}$ & FPSs $\left(\mathbf{P}_{3}\right)$ & $\left.\Pi_{3}\right)$ \\
\hline$\vdots$ & $\vdots$ & $\vdots$ \\
\hline$P_{1}$ & FPSs $\left(P_{1}\right)$ & $\Pi_{1}=$ FFPS \\
\hline
\end{tabular}

Fig. 3. The relationship of $P_{i}, \operatorname{FPSs}\left(P_{i}\right)$ and remaining UPSs $\Pi_{i}$

\section{B. Pattern Generation}

Definition 3: The set consists of all patterns with $m 1 \mathrm{~s}$ and $(N-m) 0$ s is denoted as $\Theta_{m}^{N}$, where $m \in[0,1,2, \cdots, N-1$, $N]$. The size of $\Theta_{m}^{N}$ is the number of patterns in $\Theta_{m}^{N}$ and is denoted as $\left|\Theta_{m}^{N}\right|$.

Example 3: For a 4-input core, $\Theta_{0}^{4}=\{0000\},\left|\Theta_{0}^{4}\right|=1$. $\Theta_{1}^{4}=\{1000,0100,0010,0001\},\left|\Theta_{1}^{4}\right|=4$.

Theorem 1: $\Theta_{m}^{N}$ can activate all (N!-1) POFs where $m \in[1$, $2, \cdots, N-2, N-1]$. [6]

According to Theorem 1, we arbitrarily apply one $\Theta_{m}^{N}$ to the inputs of core for $m \in[1,2, \cdots, N-2, N-1]$. Since $\left|\Theta_{m}^{N}\right|$ is smaller when $m$ is closer to the end points of interval $[1,2, \cdots$, $N$-1], we select $m$ from 1 up to $\lfloor N / 2\rfloor$ or from $N$-1 down to $\lfloor N / 2\rfloor$.

Given an 8-input combinational core, the initial UPSs $\Pi_{0}$ are (12345678). The simulation results of $\Theta_{1}^{8}$ are shown in Fig. 4 and are represented in symbolic output representation. The patterns with the same output are grouped into one set. $\Theta_{1}^{8}$ patterns can be grouped into two sets, S1 and S2. When we select the smaller set $\mathrm{S} 1$ as the verification pattern set $P_{1}, \Pi_{1}$ have to be derived as well. The following paragraphs describe how to calculate the UPSs $\Pi_{i}$ when $P_{i}$ is generated.

Definition 4: Given a set of patterns $S$ with the same length, we count the number of digits 1 in the same bit position to form a vector with the same length. This vector is called the characteristic vector $(\mathrm{CV})$ of $\mathrm{S}$ and is denoted as CV_S.

Theorem 2: A pattern set $S$ ', which consists of all same output patterns $\in \Theta_{m}^{N}$, turns to $S$ after a FPS $\lambda$. If $C V S_{-}{ }^{\prime} \neq C V-S$, then the FPS $\lambda$ will be detected by $S$ '.

In Fig. 4, we selected $\mathrm{S} 1$ as $P_{1}$, and according to Theorem 2 , any port sequence which changes CV_S1 will be detected by S1. Thus, the port misplacement that cannot change CV_S1 are regarded as the UPSs $\Pi_{1}$.

Corollary 1: If a pattern set $S$ is selected as the verification pattern set $P_{i}$, the UPSs $\Pi_{i}$ can be obtained by applying the same grouping result of CV_S over the UPSs $\Pi_{i-1}$.

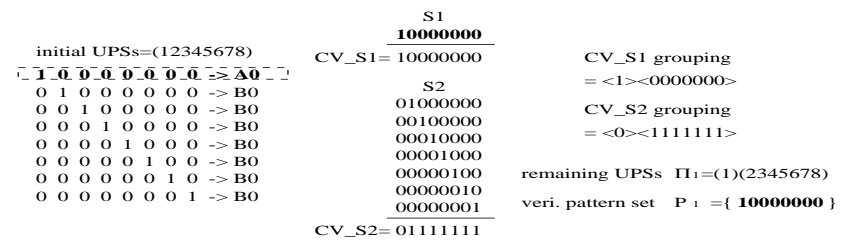

Fig. 4. The simulation outputs of $\Theta_{1}^{8}$ 
When $\mathrm{S} 1$ is selected as $P_{1}$. The grouping result of CV_S1 is $\langle 1><0000000\rangle$. Therefore, according to Corollary $1, \Pi_{1}$ is obtained by the same grouping of CV_S1 over the UPSs $\Pi_{0}=(12345678)$ directly and $\Pi_{1}$ become (1)(2345678). These results are also shown in Fig. 4. If the port sequence $\lambda$ of the real interconnection in the integrated design $\in \operatorname{FPSs}\left(P_{1}\right)$, when applying $P_{1}$ into the integrated design, $\lambda$ will be detected.

To demonstrate the interconnection detection, diagnosis, and correction procedures, we assume the FPS $\lambda$ of this example is given and is 83762451 .

\section{Fault Detection}

We apply $P_{1}\{10000000\}$ into the design with the FPS $\lambda$ 83762451 , and find that the corresponding output of $P_{1}$ is B0 as shown in the first row of Fig. 5(a). Since the fault free output is A0, thus the fault effect appears and $\lambda$ is detected by $P_{1}$.

\section{Fault Diagnosis}

We apply $P_{1}$ into the integrated design, and realize that the real applied input is not $P_{1}$ by observing the unexpected output B0. Since there are seven patterns that produce the B0 output, we do not know exactly what the actual applied pattern is. Nevertheless, we know the actual applied pattern which produces the A0 output instead. We simulate $\Theta_{1}^{8}$ patterns with the FPS 83762451 and observe the outputs until the output is A0. These results are shown in Fig. 5(a). From Fig. 5(a), we find that when the last pattern $\{0000001\}$ is the output becomes A0. This result implies that the FPS $\lambda$ turns the pattern $\{00000001\}$ to $\{10000000\}$. We put the pattern $\{00000001\}$ into S1' and assume S1 is $\{10000000\}$. Then we calculate CV_S1' $=00000001$ and CV_S1 10000000 . Afterward, the misplaced ports can be identified by comparing CV_S1' and CV_S1.

\section{E. Fault Correction}

Comparing CV_S1' and CV_S1, we observe that the $1^{\text {st }}$ digit and the $8^{t h}$ digit of CV_S1' and CV_S1 are different. Thus, we switch the port 1 with the port 8 to let CV_S1 be the same as CV_S1'. The corrected FPS becomes 13762458 and is shown in Fig. 5(b).

Definition 5: The exchange of two ports is defined as a 2_switch. The exchange of port $\mathrm{x}$ and port $\mathrm{y}$ is denoted as 2_switch(x, y).

Definition 6: Given a set of n-bit patterns S', when a 2-switch is applied on S' and CV_S' is invariant, the 2-switch is called a CV invariant fault of $S^{\prime}$ (CVIF( $\left.\left.S^{\prime}\right)\right)$. Otherwise, it is called a $\mathrm{CV}$ variant fault of $\mathrm{S}^{\prime}\left(\mathrm{CVVF}\left(\mathrm{S}^{\prime}\right)\right)$.

Theorem 3: Given a set of n-bit patterns $S$, and a FPS $\lambda$. There exists a finite sequence of 2_switches, 2_switch $1 \sim$ $2_{2}$ switch $_{l}$, to convert the FFPS into $\lambda$, and this sequence of $2 \_$switches must be in one of the following three categories:

(I): 2_switch 12 2_switch ${ }_{l}$ are all CVIFs ( $\left.S^{\prime}\right)$

(II): 2_switch ${ }_{1} \sim 2 \_s w i t c$ h $_{i}$ are CVIFs( $\left.S^{\prime}\right)$ and 2_switch ${ }_{i+1}$ $\sim 2 \_$switch are $_{\text {CVVFs }}\left(S^{\prime}\right)$ where $1 \leq i \leq l-1$

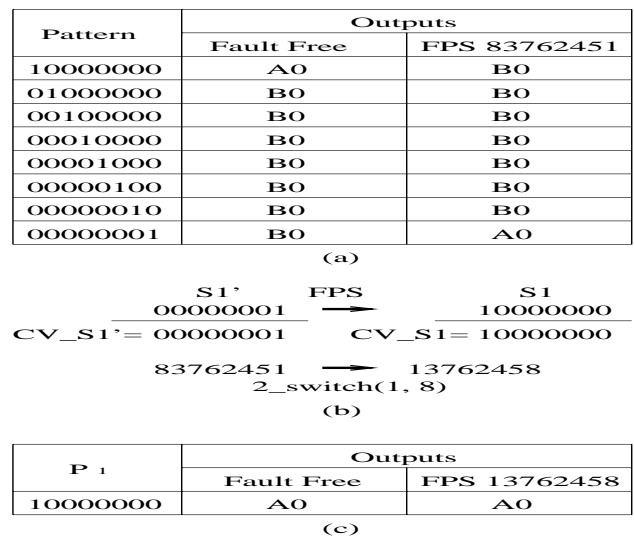

Fig. 5. Rectification processes of $\Theta_{1}^{8}$

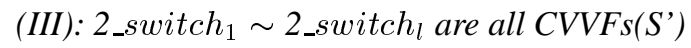

In this example, the 2_switch $(1,8)$ is a $\operatorname{CVVF}\left(\mathrm{S}^{\prime}{ }^{\prime}\right)$. Therefore, according to Theorem 3, the corrected FPS $\lambda^{\prime}$ is a sequence of CVIFs(S1'). Theorem 3 guarantees that the CVIF(S1') are the only possible faults which we have to deal with in the succeeding iteration.

Since each $P_{i}$ corresponds to a set of FPSs, $\operatorname{FPSs}\left(P_{i}\right)$, and is responsible for detecting them. If the corrected FPS $\lambda^{\prime} \notin$ $\operatorname{FPSs}\left(P_{i}\right), P_{i}$ is not able to detect and correct any other faulty ports in $\lambda^{\prime}$. At this time, further verification pattern set $P_{k}$, where $k>i$, are generated to detect and correct the other faulty ports in $\lambda^{\prime}$. However, how can we know the corrected FPS $\lambda^{\prime} \notin \operatorname{FPS}\left(P_{i}\right)$ and cannot be corrected by $P_{i}$ anymore ? The following corollary states the condition that has to be satisfied so that the corrected FPS $\lambda^{\prime} \notin \operatorname{FPSs}\left(P_{i}\right)$ (or $\in$ UPSs $\Pi_{i}$ ). Corollary 2: If the actual outputs are consistent with the expected ones when applying $P_{i}$ into the integrated design with a corrected FPS $\lambda^{\prime}$, then the corrected FPS $\lambda^{\prime} \in U P S s \Pi_{i}$.

Therefore, according to Corollary 2 , we apply $P_{1}$ into the integrated design again with the corrected FPS $\lambda^{\prime} 13762458$ to see whether the $\lambda^{\prime}$ can be further corrected by $P_{1}$. In Fig. 5(c), we find that the outputs of $P_{1}$ are both A0, thus, $\lambda^{\prime} \in$ UPSs $\Pi_{1}$ (1)(2345678). At this time, we move to the next iteration to generate further pattern sets $P_{k}(k>1)$ to detect and correct the remaining faulty ports.

\section{F. Summary}

The FPS is corrected from 83762451 to 13762458 , and the UPSs representation is reduced from (12345678) to (1)(2345678). These results illustrate that the UPSs representation presents the corrected FPS appropriately. The succeeding iterations in the AIR follow the same flow to rectify the misplaced interconnection. However, due to the page limit, we skip these repeated demonstration of the AIR algorithm here.

The success of the AIR depends on the pattern generation stage strongly. Since the pattern generation stage will search all $\Theta_{m}^{N}$, for $m=1,2, \cdots, N$-1 if necessary, it is a complete algorithm [6] [7]. This complete pattern generation algorithm leads the AIR algorithm to be complete as well. 
TABLE I

EXPERIMENTAL RESULTS ON COMBINATIONAL BENCHMARKS

\begin{tabular}{|c|c|c|c|c|c|c|}
\hline \multirow{2}{*}{ bench } & \multicolumn{2}{|c|}{ parameters } & \multicolumn{2}{c|}{ blind connection } & \multicolumn{2}{c|}{ guided connection } \\
\cline { 2 - 7 } & $|\mathrm{PI}|$ & gcs. & a/b & time & a/b & time \\
\hline$c 17$ & 5 & 6 & $5 / 5$ & 0.1 & $3 / 3$ & 0.1 \\
$c 880$ & 60 & 357 & $60 / 60$ & 182 & $13 / 13$ & 96 \\
$c 1355$ & 41 & 514 & $41 / 41$ & 179 & $8 / 8$ & 50 \\
$c 1908$ & 33 & 880 & $32 / 32$ & 167 & $6 / 6$ & 46.5 \\
$c 432$ & 36 & 160 & $36 / 36$ & 43 & $6 / 6$ & 7 \\
$c 499$ & 41 & 202 & $39 / 39$ & 59 & $9 / 9$ & 16.6 \\
$c 3540$ & 50 & 1667 & $48 / 48$ & 882 & $12 / 12$ & 268 \\
$c 5315$ & 178 & 2290 & $178 / 178$ & 11505 & $34 / 34$ & 2692 \\
$c 2670$ & 233 & 1161 & $232 / 232$ & 10119 & 3737 & 1937 \\
$c 7552$ & 207 & 3466 & $207 / 207$ & 26689 & $39 / 39$ & 6477 \\
$c 6288$ & 32 & 2416 & $32 / 32$ & 773 & $8 / 8$ & 466 \\
\hline
\end{tabular}

\section{G. The Sequential AIR}

The development of the sequential AIR is based on the same assumption as the combinational AIR, i.e., the CUV is preverified and fault free. The fault occurs only at the interconnection between the cores. For the testability concern, most sequential cores are designed with scan chains. Thus, here we assume that the sequential cores in the experiments are scantestable. These sequential cores can be set in arbitrary state and therefore they can be seen as combinational ones. Consequently, the AIR algorithm used in the combinational cores is applicable to the sequential ones. The only difference is that the sequential cores have to be set to a state by sequential AIR before evaluating outputs.

\section{EXPERIMENTAL RESULTS}

The heuristic AIR, which adds the iteration counter to bound the processing time, has been integrated into the SIS [13] environment. Experiments are conducted over a set of benchmarks, which are in BLIF format. The simulation information of the BLIF benchmarks imitate the simulation model of IP cores.

Table I summaries the experimental results of the heuristic AIR. The $|\mathrm{PI}|$ represents the number of inputs. The gate counts (gcs.) indicates the scale of a benchmark. The $a / b$ presents "number of corrected ports/number of faulty ports".

The iteration bound in the experiment was set to 100 . The AIR algorithm will be terminated automatically if the iteration counter is over the bound or the UPSs representation becomes empty. At the end of AIR, the number of corrected ports, and CPU time are returned. The number of corrected ports is obtained by comparing the final FPS with the FFPS. The CPU time is measured in second on an Ultra Sparc II workstation.

According to Table I and II, the faulty ports of each benchmark are all corrected except s5378 and s13207, and the processing time of each benchmark is acceptable. These results demonstrate that the heuristic AIR is able to correct the misplaced ports within reasonable efforts.

\section{CONCLUSIONS}

The AIR technique provides a solution to integrate the cores with correct interconnection automatically. Therefore this
TABLE II

EXPERIMENTAL RESULTS ON SEQUENTIAL BENCHMARKS

\begin{tabular}{|c|c|c|c|c|c|c|c|}
\hline \multirow{2}{*}{ bench } & \multicolumn{3}{|c|}{ parameters } & \multicolumn{1}{|c|}{ blind connection } & \multicolumn{2}{c|}{ guided connection } \\
\cline { 2 - 8 } & gcs. & FFs & a/b & time & a/b & time \\
\hline$s 1196$ & 14 & 529 & 18 & $13 / 13$ & 24 & $5 / 5$ & 14.2 \\
$s 1238$ & 14 & 508 & 18 & $13 / 13$ & 31 & $3 / 3$ & 8.9 \\
$s 1488$ & 8 & 653 & 6 & $8 / 8$ & 39 & $4 / 4$ & 30 \\
$s 1494$ & 8 & 647 & 6 & $6 / 6$ & 62 & $2 / 2$ & 41 \\
$s 15850$ & 14 & 9786 & 597 & $13 / 13$ & 1382 & $5 / 5$ & 1252 \\
$s 208$ & 11 & 104 & 8 & $11 / 11$ & 29.1 & $4 / 4$ & 21 \\
$s \mathbf{2 7}$ & 4 & 10 & 3 & $4 / 4$ & 0.1 & $2 / 2$ & 0.1 \\
$\mathbf{s 3 7 8}$ & 35 & 2779 & 164 & $\mathbf{2 9 / 3 3}$ & 3149 & $9 / 9$ & 3083 \\
$s 641$ & 35 & 379 & 19 & $35 / 35$ & 136 & $9 / 9$ & 81 \\
$s 713$ & 35 & 393 & 19 & $33 / 33$ & 137 & $7 / 7$ & 85 \\
$s 820$ & 18 & 289 & 5 & $17 / 17$ & 1005 & $5 / 5$ & 979 \\
$s 832$ & 18 & 287 & 5 & $18 / 18$ & 1014 & $8 / 8$ & 1004 \\
$s 838$ & 35 & 446 & 32 & $33 / 33$ & 1111 & $8 / 8$ & 908 \\
$s 9234$ & 36 & 5597 & 211 & $35 / 35$ & 8133 & $9 / 9$ & 7620 \\
$s 444$ & 3 & 181 & 21 & $3 / 3$ & 62 & $2 / 2$ & 56 \\
$s 510$ & 19 & 211 & 6 & $18 / 18$ & 130 & $6 / 6$ & 105 \\
$s 344$ & 9 & 160 & 15 & $9 / 9$ & 4.5 & $4 / 4$ & 2.6 \\
$s 349$ & 9 & 161 & 15 & $9 / 9$ & 7.8 & $3 / 3$ & 2.1 \\
$s 382$ & 3 & 158 & 21 & $3 / 3$ & 44 & $3 / 3$ & 44 \\
$s 386$ & 7 & 159 & 6 & $5 / 5$ & 46 & $3 / 3$ & 40 \\
$s 400$ & 3 & 162 & 21 & $3 / 3$ & 49.6 & $3 / 3$ & 46 \\
$\mathbf{s 1 3 2 0 7}$ & 31 & 8027 & 669 & $\mathbf{2 3 / 2 8}$ & 19114 & $4 / 4$ & 9352 \\
$s 1423$ & 17 & 657 & 74 & $17 / 17$ & 1586 & $5 / 5$ & 375 \\
$s 6669$ & 83 & 3080 & 239 & $83 / 83$ & 12954 & $1 / 16$ & 10223 \\
$s 4863$ & 49 & 2342 & 104 & $49 / 49$ & 7733 & $11 / 11$ & 4551 \\
$s 1269$ & 18 & 569 & 37 & $16 / 16$ & 56 & $4 / 4$ & 24.8 \\
$s 1512$ & 29 & 780 & 57 & $29 / 29$ & 2489 & $6 / 6$ & 1932 \\
$s 3271$ & 26 & 1572 & 116 & $25 / 25$ & 6254 & $7 / 7$ & 4876 \\
$s 330$ & 40 & 1789 & 132 & $40 / 40$ & 805 & $6 / 6$ & 156 \\
$s 3384$ & 43 & 1685 & 183 & $43 / 43$ & 6377 & $9 / 9$ & 4458 \\
$b 10$ & 11 & 153 & 17 & $10 / 10$ & 170 & $3 / 3$ & 157 \\
$b 11$ & 7 & 510 & 31 & $7 / 7$ & 230 & $4 / 4$ & 185 \\
$b 12$ & 5 & 880 & 121 & $5 / 5$ & 482 & $3 / 3$ & 315 \\
$b 13$ & 10 & 255 & 53 & $8 / 8$ & 226 & $3 / 3$ & 210 \\
$b 14$ & 32 & 5401 & 245 & $31 / 31$ & 16332 & $7 / 7$ & 13453 \\
$b 15$ & 37 & 7092 & 449 & $37 / 37$ & 18742 & $10 / 10$ & 16667 \\
\hline
\end{tabular}

technique can reduce the time on design verification in corebased design methodology.

\section{ACKNOWLEDGEMENTS}

This work was supported in part by ROC National Science Council under Grant NSC90-2215-E-009-106

\section{REFERENCES}

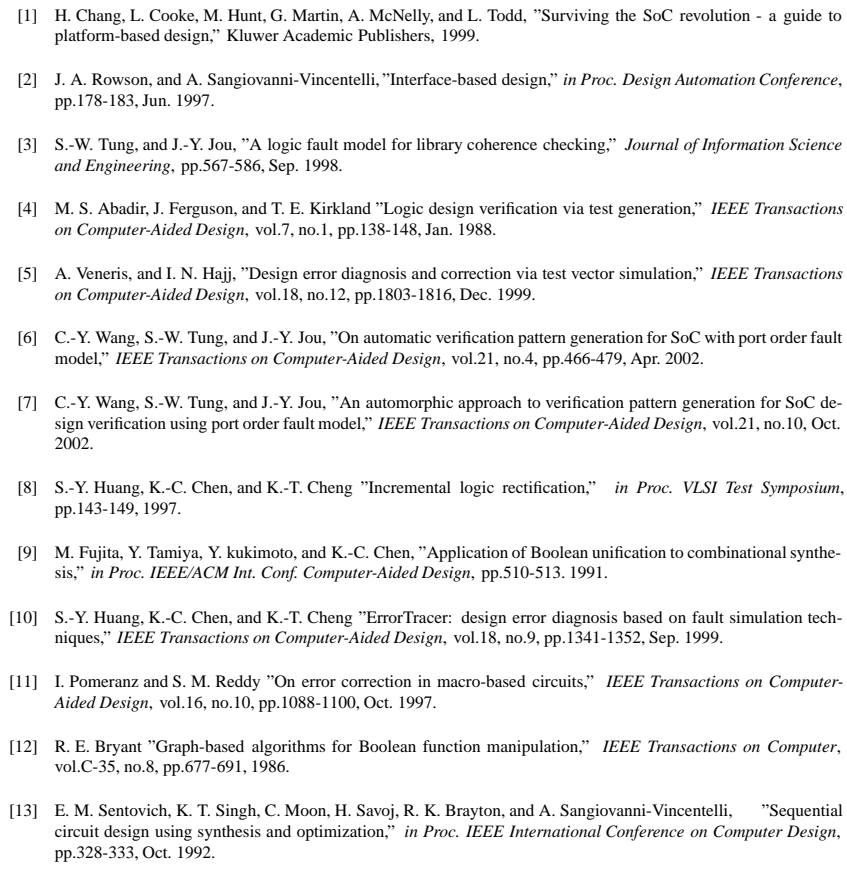
platform-based design," Kluwer Academic Publishers, 1999.

[2] J. A. Rowson, and A. Sangiovanni-Vincentelli, "Interface-based design," in Proc. Design Automation Conference, pp.178-183, Jun. 1997.

[3] S.-W. Tung, and J.-Y. Jou, "A logic fault model for library coherence checking," Journal of Information Science and Engineering, pp.567-586, Sep. 1998.

[4] M. S. Abadir, J. Ferguson, and T. E. Kirkland "Logic design verification via test generation," IEEE Transactions on Computer-Aided Design, vol.7, no.1, pp.138-148, Jan. 1988.

[5] A. Veneris, and I. N. Hajj, "Design error diagnosis and correction via test vector simulation," IEEE Transactions on Computer-Aided Design, vol.18, no.12, pp.1803-1816, Dec. 1999.

[6] C.-Y. Wang, S.-W. Tung, and J.-Y. Jou, "On automatic verification pattern generation for SoC with port order fault model," IEEE Transactions on Computer-Aided Design, vol.21, no.4, pp.466-479, Apr. 2002.

[7] C.-Y. Wang, S.-W. Tung, and J.-Y. Jou, "An automorphic approach to verification pattern generation for SoC design verification using port order fault model," IEEE Transactions on Computer-Aided Design, vol.21, no.10, Oct.

[8] S.-Y. Huang, K.-C. Chen, and K.-T. Cheng "Incremental logic rectification," in Proc. VLSI Test Symposium, pp. $143-149,1997$

[9] M. Fujita, Y. Tamiya, Y. kukimoto, and K.-C. Chen, "Application of Boolean unification to combinational synthesis," in Proc. IEEE/ACM Int. Conf. Computer-Aided Design, pp.510-513. 1991.

[10] S.-Y. Huang, K.-C. Chen, and K.-T. Cheng "ErrorTracer: design error diagnosis based on fault simulation techniques," IEEE Transactions on Computer-Aided Design, vol.18, no.9, pp.1341-1352, Sep. 1999.

[11] I. Pomeranz and S. M. Reddy "On error correction in macro-based circuits," IEEE Transactions on ComputerAided Design, vol.16, no.10, pp.1088-1100, Oct. 1997.

[12] R. E. Bryant "Graph-based algorithms for Boolean function manipulation," IEEE Transactions on Computer, vol.C-35, no.8, pp.677-691, 1986

[13] E. M. Sentovich, K. T. Singh, C. Moon, H. Savoj, R. K. Brayton, and A. Sangiovanni-Vincentelli, ＂Sequential circuit design using synthesis and optimization," in Proc. IEEE International Conference on Computer Design, pp.328-333, Oct. 1992. 